\title{
El sistema de tutorías en la Universidad. Estudio de caso de la Universidad Estatal Península de Santa Elena (UPSE)
}

\section{The system of tutoring in the University. Case study of the Península de Santa Elena State University (UPSE)}

\author{
María Caridad Mederos Machado ${ }^{1 *}$, Freddy Enrique Tigrero Suárez ${ }^{1}$, Margarita de las Nieves Lamas González $^{1}$ \\ y Néstor Vicente Acosta Lozano ${ }^{1}$ \\ ${ }^{1}$ Universidad Estatal Península de Santa Elena \\ *mmederos@upse.edu.ec
}

DOI: https://doi.org/10.26871/killkana_social.v2i4.421

\begin{abstract}
Resumen
En la Educación Superior las tutorías cobran una importancia singular, pues los elementos académicos que no quedan completos en la docencia o en la experimentación y la práctica, pueden ser aclarados y corregidos en ellas. Este trabajo tiene como objetivo describir las características actuales del sistema de tutorías que se desarrollan en la UPSE y ofrecer recomendaciones para su mejoramiento. Para ello se aplicó una encuesta a 204 profesores de la UPSE, cuyos resultados denotan que las tutorías que allí se desarrollan, responden en mayor medida a la modalidad individual y grupal, siendo escasa en línea. Las tutorías de contenidos de las asignaturas, mayoritariamente a solicitud de los estudiantes son las que más se practican en la universidad estudiada. Los estudiantes reconocen el valor de las tutorías, al mismo tiempo que los docentes consideran que han incidido positivamente en el desempeño y los resultados académicos. Las tutorías de consejería son poco desarrolladas en la UPSE, aun cuando constituyen una fuente valiosa de desarrollo humano. Se ofrecen algunas recomendaciones.
\end{abstract}

Palabras clave: profesor- tutor, tutoría académica, consejería.

\begin{abstract}
In Higher Education, the tutorials acquire a singular importance, because the academic elements that are not complete in the teaching or in the experimentation and the practice, can be clarified and corrected in them. The objective of this work is to describe the current characteristics of the tutorial system developed in the UPSE and to offer recommendations for its improvement. For this purpose, a survey was applied to 204 UPSE professors, whose results indicate that the tutorials developed there respond to a greater extent to the individual and group modality, being scarce online. The tutorials of content of the subjects, mostly at the request of students are those that are most practiced in the university studied. The students recognize the value of the tutorials, at the same time that the teachers consider that they have had a positive impact on the performance and the academic results. Counseling tutors are little developed in the UPSE, even though they constitute a valuable source of human development. Some recommendations are offered.
\end{abstract}

Key words: teacher-tutor, academic tutoring, counseling.

\section{Introducción}

Las tutorías constituyen elementos centrales en el desarrollo de la actividad docente y en el logro de competencias profesionales y humanísticas en los estudiantes de la universidad moderna. Se precisa que los profesores tutores, tengan conciencia de sus funciones y de la importancia de esta forma de conducir y estimular el conocimiento propio y de la ciencia, por parte de los estudiantes.

En el caso de la Universidad Estatal Peninsula Santa Elena (UPSE), existe un instructivo para el desarrollo de las tutorías universitarias y sin embargo, se manifiestan dificultades en esta actividad, las que en muchos casos están dadas por desconocimiento y porque los nuevos horizontes epistemológicos, son disruptivos en relación con el sistema organizacional académico que se ha estado utilizando por la universidad ecuatoriana y latinoamericana, hasta nuestros días.

En la actualidad se precisa una mayor autonomía del estudiante, dadas las características de los escenarios de aprendizaje, lo que conlleva a menos contenido y más formas de aprenderlo. $\mathrm{Y}$ en ese espacio es que cobran sentido las tutorías universitarias. 
En este contexto se desarrolla este trabajo, que tiene como objetivo describir las características actuales del sistema de tutorías que se desarrollan en la UPSE y ofrecer recomendaciones para su mejoramiento.

\section{Marco teórico}

El Sistema de Educación Superior del Ecuador a través de normativas y documentos pertinentes ha regulado procesos en la organización del aprendizaje. El Reglamento de Régimen Académico (CES, 2017b) establece el componente de docencia, correspondiente a las actividades de aprendizaje que se realiza con el acompañamiento del docente en los diferentes ambientes o entornos de aprendizaje, garantizando resultados pedagógicos.

El Consejo de Educación Superior (CES, 2017a), determina en su Reglamento de Carrera y Escalafón del Profesor e Investigador las actividades de docencia relacionadas a las tutorías académicas: a) Orientación y acompañamiento a través de tutorías presenciales o virtuales, individuales o grupales; b) Visitas de campo, tutorías (... ); c) Dirección, tutorías, seguimiento y evaluación de prácticas preprofesionales; d) Dirección y tutorías de trabajos para la obtención del título (...), todas con el fin de que los docentes de las Instituciones de Educación Superior IES se involucren en dicha labor.

La Universidad Estatal Península de Santa Elena (UPSE, 2017), presenta Instructivo Metodológico del Sistema Institucional de Tutorías de carácter sistémico e integral con la participación de los docentes en el acompañamiento académico personal, desde que el estudiante ingresa a la universidad hasta su graduación. Este servicio universitario responde a la tutoría como actividad de docencia favoreciendo las relaciones asertivas entre profesores y estudiantes.

La tutoría universitaria de la UPSE se constituye en un apoyo académico que impulsa y promueve la participación del vicerrector, comisiones de tutorías de facultades, responsables de tutorías de carreas, profesores tutores, tutorados y tutores en atención especial; en cada unidad de organización curricular los tutores realizan un trabajo de estrategias de estudio y de trabajo independiente en la solución de problemas profesionales, desarrollo de habilidades investigativas, en la orientación de prácticas preprofesionales, actividades de vinculación, al servicio de apoyo al trabajo científico y de investigación.

Para ello, existen modalidades de tutoría grupal, tutoría individual, tutoría de atención especial y tutoría en línea, cuyos roles que desempeñan los tutores son eficaces en el inicio, durante y al final del proceso formativo de los tutorados en asuntos relacionados con su desempeño académico y personal.

Las tutorías académicas o tutorías como actividad de docencia permite mediar la formación integral de los estudiantes, favorecer el análisis y la solución de las problemáticas identificadas en el proceso educativo entre los responsables de las tutorías, a continuación se detallan los siguientes tipos de tutorías académicas: tutoría de asignatura, tutoría de prácticas preprofesionales, tutorías de proyectos de servicio social y tutorías de trabajos de graduación.

Según Rodríguez (2004), citado por (Tigrero, Cedeño, y Núñez, 2017), la tutoría universitaria se desarrolla en los ámbitos académico, personal y profesional, donde las actividades tutorales se centran en coadyuvar en el dominio de los conocimientos, incluyendo orientación para que los estudiantes se capaciten en la profesión y se ajusten a las necesidades del mercado laboral. Por lo tanto, los docentes deben establecer los andamiajes para las respectivas tutorías.

Para (Tejeda-Rodríguez, 2016), "La Tutoría Académica en educación superior, es considerada como una herramienta para la construcción guiada del aprendizaje de los estudiantes así como para lograr desarrollar su autonomía y contribuir al desarrollo integral de las personas", es decir que el tutor orienta, asesora y acompaña a los tutorados para la mejora continua.

\section{Resultados y discusión}

Se realizó un estudio descriptivo, y un procesamiento estadístico sencillo, de análisis porcentual de las respuestas de una encuesta aplicada a una muestra de 204 profesores de la UPSE. La misma se estructuró en torno a las modalidades y tipos de tutorías, a la cantidad de horas dedicadas a las mismas, a los motivos por los que se ofrecen, a la valoración que ofrecen los estudiantes a las mismas y a los problemas que con mayor frecuencia se atienden en ellas.

La muestra estuvo integrada por el $43,1 \%$ de mujeres y $56,9 \%$ de hombres, donde la mayoría de ellos trabaja por igual en las Unidades de Organización Curricular Básica y Profesional, sólo menos de una cuarta parte de ellos se dedica a la Unidad de Titulación.

Ante la pregunta de si ha sido informado como tutor, se aprecia que el $28,9 \%$ no recuerda, e igual cantidad dicen que sí. Un 24,5 \% dice que no y el 18,6 plantea que sólo en períodos cercanos a los exámenes. Esta situación evidencia la escasa concienciación entre los directivos de las unidades académicas, a la hora de orientar a los docentes, en las actividades que debe desarrollar y cómo hacer las mismas. Al mismo tiempo limita el alcance que pueden tener las tutorías.

Esta información puede ser relacionada con las respuestas a la preguntas, de si realizan tutorías o no, donde el $35 \%$ asegura no haber realizado tutorías con los estudiantes. De modo que aparentemente, queda incompleta la función de orientación para el aprendizaje autónomo o la ayuda en los problemas que aunque no sean académicos, pueden afectar el rendimiento docente.

Suelen trabajarse tutorías Grupales, Individuales y En línea. Si se comparan estas modalidades por facultad, encontramos que la tendencia es trabajar más la tutoría individual, seguido de la grupal y muy poco la que se realiza en línea. (Figura 1) 


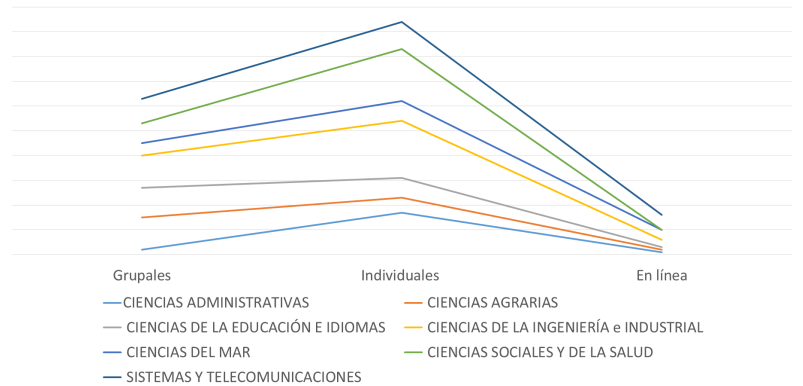

Figura 1. Tendencias en las modalidades de tutorías

Las tutorías que más se realizan por los docentes encuestados son las relacionadas con la asignatura que imparten (44\%), seguidas de las que orientan la práctica preprofesional $(32,4 \%)$, después las que corresponden a la unidad de titulación y por último a las de consejería, con el $4,9 \%$. Figura 2. Se presume que esta última es poco utilizada, porque al encontrarse atención especializada en psicología educativa y clínica, en el área de Bienestar estudiantil, los estudiantes acuden a estos y no a los docentes, sin considerar que los docentes pueden ofrecer consejos generales que sirvan de base para su proyecto de vida y a la solución de problemas sencillos de la cotidianeidad juvenil.

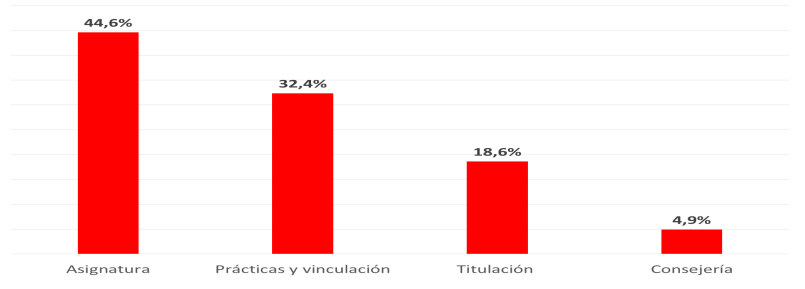

Figura 2. Tipos de tutorías usadas

En relación al tiempo semanal que se dedica a las tutorías, se conoce que el $35 \%$ dedica entre 3 y 4 horas, el $32 \%$ más de 4 horas y el resto menos de 3 horas a la semana para las actividades correspondientes, con independencia o no de haber sido informados de la tutoría.

Las motivaciones para la tutoría están vinculadas en el $48,5 \%$ a las solicitudes que los estudiantes realizan a los docentes, el $26,5 \%$ refiere que sus tutorías responden a las necesidades propias de la asignatura que imparten, mientras que solo el $14 \%$ las realiza a partir de su planificación académica. (Figura 3).

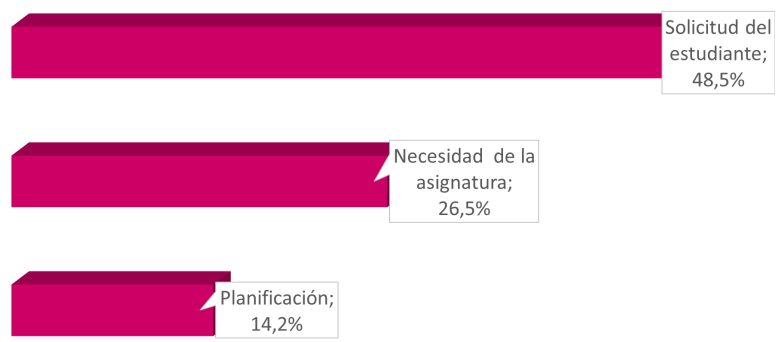

Figura 3. Motivos de la tutoría

Las valoraciones que hacen los estudiantes de las tutorías académicas, indican que son de calidad y que sienten utilidad, según indica el Figura 4. Sólo el 8,8\% refiere no ver valor alguno en las tutorías.

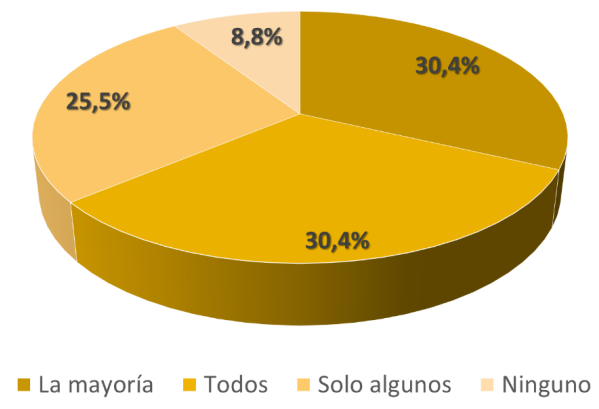

Figura 4. Valor que otorgan los estudiantes a las tutorías

Esta información puede ser relacionada con los resultados obtenidos en la pregunta ¿La tutoría ha mejorado el desempeño estudiantil? Los encuestados $(45,6 \%)$ respondieron que siempre y el 33,3\% dijo que casi siempre.

Los problemas más atendidos en las tutorías son los académicos, seguidos de los personales y otros significan solo un 3,9\% en la encuesta realizada.

Entre las consideraciones realizadas por los docentes encontramos que:

- La carga horaria y la participación en comisiones dificulta las tutorías. Deberían estar señaladas en el distributivo.

- Deberían gestionarse tutorías virtuales.

- Los informes de planificación de tutorías se solicitan desde el primer ciclo, lo que no debería ser así, pues se puede evaluar al estudiante al finalizar primer ciclo y seleccionar a los que deben asistir a tutorías por su rendimiento académico.

- Falta de área o espacio para brindar las tutorías.

- Sería bueno tener menos de 24 horas de clases para dar tutorías con mejor preparación y más significativas y con mejores resultados. 
- Las tutorías deben ser de acuerdo a lo que el estudiante considere una debilidad y no necesariamente desarrollarlas con el mismo docente.

- Se debe considerar que la universidad tenga un centro de redacción científica

\section{Conclusiones}

Las tutorías que se desarrollan en la UPSE, responden a las modalidades, individual, grupal y en línea, siendo la primera la de mayor uso.

Las tutorías de contenidos de las asignaturas, realizadas mayoritariamente a solicitud de los estudiantes son las que más se practican en la universidad estudiada.

Los estudiantes reconocen el valor de las tutorías, al mismo tiempo que los docentes consideran que han incidido positivamente en el desempeño y los resultados académicos.

Las tutorías de consejería son poco desarrolladas en la UPSE, aun cuando constituyen una fuente valiosa de desarrollo humano.

\section{Recomendaciones}

1) Priorizar la tutoría en línea. Para ello deben establecerse como metas mejores posibilidades desde la infraestructura informática, la capacitación del docente y lo más importante la concientización de la importancia de la misma, por su efectividad académica y el ahorro de tiempo.

2) Las tutorías académicas, deben orientarse y desarrollarse de modo que permitan:

- Hacer a los estudiantes más efectivos, independientes y reflexivos (análisis de sus posibilidades y limitaciones).

- Facilitar el progreso hacia el logro de los objetivos personales.

- Mejorar las habilidades de estudio y "gestión de la carrera"

- Ampliar el "universo" del aprendizaje (académico y no académico). -

- Aprender a comunicarse con otros.

- Incentivar el potencial personal

3) Las tutorías de consejería deben poner énfasis en:

- Promover una relación personal que fomente el diálogo tutor-alumno.

- Ofrecer una preparación y formación especializada al profesor para el desempeño de su función como tutor.
- Apoyar y reconocer institucionalmente la labor como tutor.

- Consolidar la figura del profesor no sólo como orientador y motivador del conocimiento sino también como promotor de la formación integral del alumno.

- La integración social y académica del alumno, fomentando tanto las relaciones formales como las informales.

\section{Referencias Bibliográficas}

CES. (2017a). Reglamento de carrera y escalafón del profesor e investigador. educación superior. Quito.

CES. (2017b). Reglamento de régimen académico. Quito: CErbarbS.

Tejeda-Rodríguez, M. (2016). La tutoría académica en el proceso de formación docente. opcion, 32(13), 879899. Descargado de https://www.redalyc . org/pdf/310/31048483042.pdf

Tigrero, F., Cedeño, J., y Núñez, L. (2017). Sistema de orientación y acompañamiento pedagógicoandragógico de tutorías académicas para la carrera de administración de empresas, universidad estatal península de santa elena. Revista de Ciencias Pedagógicas e Innovación, 5(2), 7-11. Descargado de https://incyt.upse.edu.ec/ pedagogia/revistas/index.php/rcpi/ article/view/168 doi: https://doi.org/ 10.26423/rcpi.v5i2.168

UPSE. (2017). Instructivo metodológico del sistema institucional de tutorías. Santa Elena.

Recibido: 15 de septiembre de 2018

Aceptado: 25 de noviembre de 2018 